\title{
Mitigation of transonic shock buffet on a supercritical aerofoil through wavy leading edges
}

\author{
Enrico Degregori ${ }^{1, a)}$ and Jae Wook Kim (김재욱) ${ }^{1, b}$ ) \\ Institute of Sound and Vibration Research, University of Southampton, Southampton, SO17 1BJ, \\ United Kingdom
}

(Dated: 29 December 2020)

Mitigation of shock buffet phenomenon over a supercritical aerofoil by means of wavy leading edges (WLEs) is analysed with implicit large eddy simulations (ILES). A Dassault Aviation's V2C aerofoil is simulated in a transonic flow with $R e_{\infty}=5.0 \times 10^{5}$ and $M_{\infty}=0.7$ at $\alpha=7.0^{\circ}$. This aerofoil profile is designed for transonic flows, delaying the onset of wave drag and decreasing the skin friction drag. The results of this upstream flow condition on a straight aerofoil is a large oscillation of lift and drag coefficients. In the first part, timeaveraged aerodynamic characteristics over a straight leading edge (SLE) aerofoil and three modified aerofoils with different wavy amplitudes are compared. The results show that overall WLE aerofoils are more efficient than SLE ones and the aerofoil with the lowest amplitude $(h=0.0075)$ is the most efficient, increasing lift coefficient and decreasing drag coefficient. The flow unsteadiness has a key role for aerofoils in transonic flows at moderate and high angles of attack. Hence, the second part of the paper is a detailed unsteady analysis of flow phenomena. The starting point is an investigation of unsteady aerodynamic performance. It is observed that WLE aerofoils are capable of significantly decreasing low-frequency oscillations' amplitude, identified with shock buffet. The best performance is obtained with $h=0.0125$ where a high-frequency oscillation becomes the dominant unsteady phenomenon. High-frequency oscillations are identified through the application of a frequency filtering method to the flow field. It is proved that the oscillation on the SLE aerofoil is related to vortex shedding, while the one on WLE aerofoils is related to laminar separation bubble (LSB) breathing. Overall, the unsteady analysis shows a connection between shock buffet and LSB breathing phenomena on wavy aerofoils and identifies the WLE amplitude as a key parameter to control this relation and mitigate the shock buffet.

\section{INTRODUCTION}

Transonic flow regime represents a huge challenge for aeronautical industry in terms of fuel consumption, fluidstructure interaction and noise emission due to the drag divergence which decreases the wing efficiency and the shock wave oscillations which generate an unsteady loading. Supercritical aerofoils have been designed to tackle these issues, they can be found in commercial aviation aircrafts wings, helicopters rotor blades and compressor blades. In addition, control devices have been studied to improve the aerodynamic performance and mitigate the unsteady phenomena, they can be classified into three main categories: trailing edge devices (TEDs), vortex generators and shock control bumps.

A common feature among the classic models describing the shock buffet phenomenon is the coupling between large scales excursions of the shock and pressure fluctuations at the trailing edge (TE). Static trailing edge flaps have been designed to control this large oscillations, the flap deflection modifies the flow field and stabilises it. It has been observed that trailing edge flaps can significantly diminish the buffet lift response in transonic flows and thus control the aerodynamic coefficients unsteadiness 1 . The main drawback is a drag increase which diminishes the aerofoil efficiency together with longitudinal instability, the solution is to apply a feedback loop control ${ }^{2}$. Open loop static TEDs deflections are effective in delaying the onset of shock buffet phenomenon, but they are not effective in controlling the magnitude of pressure fluctuations once the shock buffet phenomenon is fully developed. The efficacy of this device is increased with close loop control, but the performance decreases with three dimensional wing-fuselage models 3 .

Mechanical and fluidic vortex generators (VGs) have been studied extensively to understand their effect on shock wave boundary layer interaction (SBLI), which is the driving phenomenon of shock oscillations 4 . 6 . It has been observed that they energise and stabilise the boundary layer, as a consequence the flow tends to be attached to the aerofoil and the shock induced separation is inhibited. Previous studies ${ }^{7}$ show that VGs are able to increase the aerodynamic performance at the buffet onset condition, but they can significantly increase the drag at lower angles of attack. It

\footnotetext{
a) Electronic mail: E.Degregori@soton.ac.uk

b) Electronic mail: j.w.kim@soton.ac.uk
} 
is observed that the shock wave moves downstream over the modified aerofoil and the effect can be enhanced with

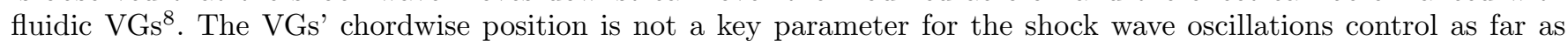
they are located upstream of the SBLI region 9 . The location of VGs becomes important on a three dimensional wing with a sweep angle due to cross flow additional components10|11. Fluidic vortex generators have been studied in an open loop configuration with a continuous or a pulsed approach. In the first case, the main parameter of the control device is the mass flow rate and its effect saturates above a threshold $\frac{8}{}$. In the second case, the main parameter is the forcing frequency. Low frequency pulsing results in shock smearing, while high frequency pulsing stabilises the shock and locates it upstream relative to the continuous fluidic blowing.

Two-dimensional shock control bumps (SCBs) were originally studied for pressure wave drag reduction $\frac{12[13}{\text { because }}$ they allow to split a normal shock into a $\lambda$-shape shock and, in this way, the total pressure losses can be decreased. The main issue with this control device is the performance in off-design conditions. An upstream movement of the shock wave leads to the generation of a second normal shock downstream by the bump, while a downstream movement of the shock wave results in BL separation and thickening. Experimental studies ${ }^{13}$ have shown that a sweep angle has a limited influence on the bump effectiveness, both pressure distribution and BL development are similar over an aerofoil and a swept wing. However, the drag reduction is slightly lower over a swept wing because of the lower contribution of wave drag to the total drag. Three-dimensional SCBs have the same working principle as the twodimensional counterpart for wave drag reduction ${ }^{14}$. A good performance is observed over unswept wings, while the efficiency is decreased over swept wings 15 .

Shock control bumps have been recently studied to control the shock buffet phenomenon $16 \mid 17$. Two-dimensional devices are able to delay the shock buffet onset modifying the flow characteristics between the shock wave and the trailing edge of a supercritical aerofoil and postponing the flow breakdown $\frac{18}{1}$. SCBs location is an important parameter, they should be placed between the shock wave and the wing trailing edge in order to mitigate the unsteady phenomenon, but in this way, they do not bring to any drag benefit. Three-dimensional bumps applied to shock oscillation control have a different working principle, they generate two streamwise vortices adjacent to the bump which energise the BL. They operate as 'smart' vortex generators ${ }^{17}$, thus the design location is upstream of the SBLI region. Their performance is still not well defined; previous studies show that they can suppress the shock oscillation $\frac{16}{6}$ or decrease the angle of buffet onset 19 . The main drawback is that the optimal position for wave drag control is different from the one for shock buffet control. A trade-off is needed to improve the overall mean and unsteady characteristics of an aerofoil over a wide range of incidence angles 17 .

Wavy leading edges can be classified as 'smart' vortex generators because they generate counter-rotating streamwise vortices at the trough 2021 , without introducing a discontinuity in the LE geometry. It has been observed ${ }^{222}$ that streamwise BL streaks are able to attenuate disturbances and delay the laminar-turbulent transition. This can have a beneficial effect on supercritical aerofoils which are designed to decrease the skin friction drag through the extended region of laminar BL and to improve the aerodynamic characteristics in transonic flows. Previous applications of this passive technique are mainly focused on low-speed flows, usually $M_{\infty} \leq 0.3$. WLE or tubercles aerofoils have been mainly analysed at high incidence angles, with the objective to improve the aerofoil efficiency in the post-stall regime. An important feature of wavy geometry, which makes it suitable for high-speed flows, is the generation of counterrotating streamwise vortices from the trough area 21/23 25. The geometric characteristics of the wavy leading edge control these vortical structures 21 . WLE wavelength $\lambda$ defines the interaction between vortices, while WLE amplitude $h$ is comparable to the vortex generator's height. The main parameter to characterise vortical structures is the local sweep angle $h / \lambda$. A recent study ${ }^{201}$ has analysed the unsteady aerodynamic characteristics of a wavy aerofoil and it has been observed that this passive technique is able to reduce the unsteady fluctuations of aerodynamic forces in the post-stall regime. In addition, it has been shown a rapid broadening and decaying of turbulent energy spectra in the leading-edge vortex dynamics on wavy geometry. This means that WLEs can be used to control unsteady loads in subsonic flows at high angles of attack.

The present work aims to apply for the first time WLEs to mitigate the shock buffet phenomenon over a supercritical aerofoil in transonic flow. A recent study ${ }^{26}$ has shown that wavy aerofoils increase the aerodynamic efficiency at low and moderate angles of attack, mainly because they are able to decrease the pressure drag. The results of this preliminary analysis prove that WLEs in transonic flows should be analysed in further detail. The first question is, how does WLE amplitude influence the aerodynamic performance of modified aerofoils? Three different configurations are considered in order to answer this question, one with a low WLE amplitude $(h=0.0075)$, one with a conventional amplitude $(h=0.0125)$, and one with a high amplitude $(h=0.0250)$, they are called WLE1, WLE2, and WLE3, respectively. They are analysed at $\alpha=7.0^{\circ}$ in terms of both mean and unsteady aerodynamic performance. The unsteady aerodynamic performance is a key analysis considering the imposed angle of attack (AoA). One of the main research topics in high-speed flows is the mitigation of unsteady oscillations which in transonic flows results in shock buffet. This raises the second important question and justifies the choice of the imposed incidence angle, what is the influence of leading edge (LE) waviness over shock buffet phenomenon? The unsteady pressure field is analysed in detail trying to understand its trend on both straight and wavy aerofoils. Then, a frequency filtering approach on the 
flow field is applied, trying to characterise all unsteady phenomena.

This paper is structured in the following order. $\$$ II introduces the computational set-up and methods used in this study. $\llbracket$ III provides the details of time-averaged flow characteristics over the aerofoils, comparing streamwise and spanwise aerodynamic performance with previous results $\stackrel{26}{26}$ and analysing WLE amplitude effect on aerodynamic performance. $₫[\mathrm{IV}$ shows the unsteady analysis' results, both in terms of the WLE amplitude effect and the characterisation of unsteady phenomena. Finally, concluding remarks are given in $4 \mathrm{~V}$

\section{PROBLEM DESCRIPTION AND METHODOLOGY}

The aerofoil section considered in the present study is Dassault Aviation's V2C profile that has been previously used to investigate the shock buffet phenomenon 27/28. A supercritical aerofoil profile is designed for transonic flows, the flatness of the upper surface allows to delay the drag increase caused by the wave drag component. In addition, this profile is designed to decrease the skin friction drag thanks to the large regions of laminar boundary layer on both the upper and lower surfaces. The application of WLEs in transonic flows should be studied using a baseline aerofoil which is designed for these flow conditions, for this reason the Dassault Aviation's V2C profile is used in this work. The chord-based Reynolds number and the Mach number are set to $R e_{\infty}=5.0 \times 10^{5}$ and $M_{\infty}=0.7$, respectively, as previously studied in literature 28 . The angle of attack (AoA) is set to $\alpha=7^{\circ}$. The current computational study employs a high-resolution implicit large-eddy simulation method based on a wavenumber-optimised discrete filter. The filter is applied directly to the conservative variables at every time step, enforcing the dissipation of scales smaller than the filter cut-off wavelength. ILES technique was compared with an explicit SGS mode 29$]$ and, with a proper grid resolution, ILES simulations are capable of correctly capturing the flow physics.

\section{A. Governing equations and numerical methods}

The full compressible Navier-Stokes equations (non-dimensionalised) are numerically solved in a generalised coordinates system

$$
\frac{\partial}{\partial t}\left(\frac{\mathbf{Q}}{J}\right)+\frac{\partial}{\partial \xi_{i}}\left(\frac{\mathbf{E}_{j}}{J} \frac{\partial \xi_{i}}{\partial x_{j}}\right)=\frac{M_{\infty}}{R e_{\infty}} \frac{\partial}{\partial \xi_{i}}\left(\frac{\mathbf{F}_{j}}{J} \frac{\partial \xi_{i}}{\partial x_{j}}\right)-\frac{\mathbf{S}}{J}
$$

for $i=1,2,3$; and, $j=1,2,3$. The Reynolds number is $R e_{\infty}=\rho_{\infty} U_{\infty} L_{c} / \infty$ based on the mean chord length $\left(L_{c}\right)$ and the free-stream velocity $\left(U_{\infty}=\sqrt{u_{\infty}^{2}+v_{\infty}^{2}+w_{\infty}^{2}}\right)$. The Mach number is $M_{\infty}=U_{\infty} / a_{\infty}$ where $a_{\infty}=\sqrt{\gamma p_{\infty} / \rho_{\infty}}$ is the ambient speed of sound. The constituting terms in Equation 1 are given as follow:

$$
\left\{\begin{array}{l}
\mathbf{Q}=\left[\rho, \rho u, \rho v, \rho w, \rho e_{t}\right]^{T} \\
\mathbf{E}_{j}=\left[\rho u_{j},\left(\rho u u_{j}+\delta_{1 j} p\right),\left(\rho v u_{j}+\delta_{2 j} p\right),\left(\rho w u_{j}+\delta_{3 j} p\right),\left(\rho e_{t}+p\right) u_{j}\right]^{T} \\
\mathbf{F}_{j}=\left[0, \tau_{1 j}, \tau_{2 j}, \tau_{3 j}, u_{i} \tau_{i j}+q_{i}\right]^{T} \\
\tau_{i j}=\mu\left(\frac{\partial u_{i}}{\partial x_{j}}+\frac{\partial u_{j}}{\partial x_{i}}-\frac{2}{3} \delta_{i j} \frac{\partial u_{i}}{\partial x_{i}}\right) \quad q_{j}=\frac{\mu}{(\gamma-1) P r} \frac{\partial T}{\partial x_{j}}
\end{array}\right.
$$

where $x_{j}=\{x, y, z\}$ and $\xi_{i}=\{\xi, \eta, \zeta\}$ stand for Cartesian and generalised coordinates, respectively. The Jacobian determinant of the trasformation from Cartesian coordinates to body fitted ones is given by $J^{-1}=|\partial(\xi, \eta, \zeta) / \partial(x, y, z)|^{30}$. The vector $\mathbf{Q}$ contains the conservative variables where $u_{j}=\{u, v, w\}$ are the velocity components and $e_{t}=$ $p /[(\gamma-1) \rho]+u_{j} u_{j} / 2$ is the total energy. The heat capacity ratio is $\gamma=1.4$ and the Prandtl number is $\operatorname{Pr}=0.71$ for air. The convective, viscous and heat fluxes are represented by $\mathbf{E}_{j}, \mathbf{F}_{j}$ and $q_{j}$, respectively, and $\tau_{i j}$ is the stress tensor. The extra source term $\mathbf{S}$ on the right-hand side of Equation 1 is non-zero within a prescribed sponge layer ${ }^{31}$ (Figure 17), it is intended to provide a perfectly anechoic condition suppressing numerical reflections of pressure waves from the outer boundaries.

The length scales are normalised by $L_{c}$, time scales by $L_{c} / a_{\infty}$, velocities by $a_{\infty}$ and pressure by $\rho_{\infty} a_{\infty}^{2}$. Moreover, the density, temperature and viscosity are normalised by their own ambient values $\rho_{\infty}, T_{\infty}$ and $\mu_{\infty}$, respectively.

The upstream flow is two dimensional, $u_{\infty}=U_{\infty} \cos \alpha, v_{\infty}=U_{\infty} \sin \alpha$ and $w_{\infty}=0$ with the aerofoil mean chord parallel to the $x$-axis. At the beginning of the simulation, the flow is gradually accelerated from zero to the desired speed using a moving frame technique for 5 time units trough a ramping function: $\mathbf{u}_{r a m p}=\mathbf{u}_{\infty} \sin ^{2}\left[(\pi / 2) \min \left(t / t_{0}, 1\right)\right]$ where $t_{0}=5$.

The governing equations are numerically solved with high-order accurate numerical methods on a structured multiblock grid system. The flux derivatives calculation is based on eighth-order pentadiagonal compact finite difference 
schemes with five-point stencils 32 . Numerical stability is preserved applying a sixth-order pentadiagonal compact filter with a cutoff wavenumber (normalised by the grid spacing) equal to $0.7 \pi$. 33 . An adaptive nonlinear artificial dissipation model is used to increase numerical stability close to the shock wave avoiding unnecessary damping on smooth linear acoustic waves 34 . Explicit time advancing of numerical solution is carried out using the classical fourthorder Runge-Kutta scheme with a CFL number of 0.95. Characteristic non reflecting boundary conditions 35 are coupled with a sponge layer in far field boundaries. Periodic conditions are used across the spanwise boundary planes and characteristic interface conditions are imposed at the boundaries that separate two blocks. No-slip wall boundary conditions are implemented on the aerofoil surface 36 . The code is fully parallelised via domain decomposition and message passing interface (MPI) libraries, where the distributed solution of pentadiagonal matrix systems is achieved with a quasi-disjoint approach $[37$.

\section{B. Geometry and discretisation of the problem}

The computational domain is shown in Figure 1 , where the dashed line separates the physical domain from the sponge layer. A structured multi-block grid is used based on a H-topology, stretched in both streamwise and vertical directions. A very fine grid spacing is imposed close to block boundaries to avoid loss of accuracy due to boundary compact schemes (Figure 1 c). Figure $1 \mathrm{~b}$ shows that the grid cells are clustered vertically in the region $y= \pm 0.2$ in order to capture properly the interaction between the shock wave and the boundary layer.

The aerofoil geometry is given by

$$
\left\{\begin{array}{l}
x_{B}(z)=k_{w}(z)\left(x_{A}-x_{T E}\right)+x_{T E} \\
y_{B}(z)=k_{w}(z)\left(y_{A}-y_{T E}\right)+y_{T E}
\end{array}\right.
$$

where

$$
k_{w}=1-\frac{h_{L E}}{L_{c}} \sin \left[\frac{2 \pi\left(z-z_{0}\right)}{\lambda_{L E}}\right]
$$

where $\left(x_{A}, y_{A}\right)$ represents the coordinates of the straight aerofoil section and $\left(x_{B}, y_{B}\right)$ the corresponding coordinates of the wavy aerofoil section varying with the spanwise coordinate. The spanwise domain size is denoted by $L_{z}$, and it is set to 0.05. In this work, the WLE wavelength is $\lambda=0.05$, thus only one wavelength is considered, while three different amplitudes $\left(h=h_{L E}\right)$ are analysed $h=0.0075, h=0.0125$ and $h=0.0250$ which correspond to an aspect ratio of the wavy geometry $(2 h / \lambda)$ of $0.3,0.5$ and 1 , respectively. Figure 1 d shows the three dimensional wavy geometry and the mesh on the aerofoil surface. The waviness at the leading edge stretches the grid cells, and thus, the computational cost increases. In particular, it is observed that an aspect ratio equal to 1 doubles the computational cost of the simulation compared with the baseline case. The aerofoil is placed at the centre of the domain with the origin at the mid-chord location. The domain size is $x \in[-7,12]$ in the streamwise direction, $y \in[-12,12]$ in the vertical direction, and $z \in\left[-L_{z} / 2, L_{z} / 2\right]$ in the spanwise direction.

The grid is validated with three different grids on the straight aerofoil, they are reported in Table I. G1 is the grid used for the following analysis and it is consistent with a previous study 26 . Since no turbulence model is included, a sufficiently high level of near-wall grid resolution is maintained in order to properly resolve the boundary layers throughout the surface. The distribution of the mean wall variable in the normal direction is always below 1 . In the streamwise direction it is between the direct numerical simulation (DNS) and the large eddy simulation (LES) range ${ }^{29}$, while in the spanwise direction it is in the low LES range. G2 is a grid refined in the streamwise direction increasing the number of cells on the aerofoil, while G3 is a grid refined in the spanwise direction.

The time and spanwise averaged pressure $\left(C_{p}\right)$ and skin friction $\left(C_{f}\right)$ coefficients obtained with the grids in Table $\mathrm{I}$ are compared in Figure $2 \mathrm{a}$ and $2 \mathrm{~b}$. For both variables, there is a close agreement, demonstrating a reasonable level of grid convergence. Since in this study there is a large unsteady analysis, Figure 2a and 2 display a comparison of lift coefficient time signal and PSD with respect to the Strouhal number (St). The PSD shows a close agreement in the two maxima and the decay at high frequencies. The largest difference is in the decay between the first and the second peak, but the three results are consistent. Table II] shows the overall aerodynamic characteristics which confirm the possibility to use G1 grid for the following study.

Due to the imposed Reynolds number, the time step has an order of magnitude of $10^{-5}$. The computational cost is 2 wall-clock hours per time unit using 2592 processor cores in the UK National Supercomputer ARCHER, which brings to a max of 26,500 points per processor. 



FIG. 1: Computational domain highlighting physical domain and sponge layer (a), close up near the aerofoil and the wake with $25 \%$ of points shown and the streamwise velocity flow field (b), close up near the aerofoil with $25 \%$ of points shown (c) and mesh over the surface of the wavy aerofoil (repeated 5 times) with $50 \%$ of the points shown (d).

\begin{tabular}{cccccc}
\hline & $n_{\xi A E R O}$ & $n_{\xi}$ & $n_{\eta}$ & $n_{\zeta}$ & $n_{\text {tot }} / 10^{6}$ \\
\hline G1 & 800 & 1430 & 800 & 50 & 57.2 \\
G2 & 1000 & 1630 & 800 & 50 & 65.2 \\
G3 & 800 & 1430 & 800 & 75 & 85.8 \\
\hline
\end{tabular}

TABLE I: Details of grids used for validation of SLE aerofoil simulation at $\alpha=7.0^{\circ}$.

\begin{tabular}{cccc}
\hline & $\mathrm{G} 1$ & $\mathrm{G} 2$ & $\mathrm{G} 3$ \\
\hline$C_{L}$ & 0.943 & 0.931 & 0.936 \\
$C_{D}$ & 0.084 & 0.089 & 0.088 \\
$C_{L} / C_{D}$ & 11.23 & 10.46 & 10.63 \\
\hline
\end{tabular}

TABLE II: Mean lift, drag and efficiency obtained during the grid validation for SLE aerofoil.

\section{TIME-AVERAGED CHARACTERISTICS}

The first part of this study analyses the mean aerodynamic characteristics of wavy aerofoils, comparing them with the SLE counterpart. The objective is to define the effect of WLE amplitude on mean aerodynamic performance and determine the most efficient configuration before extending the analysis to unsteady characteristics. The mean aerodynamic performance is first evaluated in the streamwise direction and then in the spanwise direction, comparing the results between straight and wavy aerofoils.

\section{A. Streamwise aerodynamic performance details}

Table III shows the mean aerodynamic performance of straight and wavy aerofoils. The mean lift generated by modified aerofoils is increased with $h=0.0075$ and it tends to decrease when WLE amplitude is enhanced. As a result, WLE3 aerofoil has a lower mean lift than SLE one. The mean drag trend is different. The wavy aerofoil tends to decrease the drag coefficient and it is quite constant when WLE amplitude is increased. In order to understand this trend, it is necessary to investigate the drag components. The pressure drag on wavy aerofoils is decreased compared to the one on the baseline case and it seems to decrease when the amplitude is enhanced, while the skin friction drag on wavy aerofoils is increased compared with the one on the baseline case and it grows when the amplitude is 



FIG. 2: Grid validation for SLE aerofoil. Comparison of mean aerodynamic characteristics, mean pressure coefficient distribution (a) and mean skin friction coefficient distribution (b). Comparison of unsteady aerodynamic characteristics, lift coefficient time signal (c) and lift coefficient PSD with respect to the Strouhal number (d).

increased. The pressure drag is the dominant component in transonic flows and this explains the overall improved aerodynamic performance on wavy aerofoils. Increasing the amplitude, the effects on pressure and skin friction drag components seem to cancel each other. The trend of drag coefficient is expected to change when the amplitude is enhanced to values higher than the ones considered in this study. In this case, the drag should start increasing because of the increased flow distortion at the LE which should bring to early separation.

Figure 3 shows the pressure coefficient over the main sections (peak, hill, and trough) of the three wavy aerofoils and compares it with the distribution over the straight one. The pressure distribution shape over the upper surface changes between straight and wavy aerofoils. The mean pressure coefficient on the baseline aerofoil increases regularly from the LE moving downstream up to the TE. This is a sign of large and periodic shock wave oscillations over time. On the other hand, the mean pressure coefficient over the modified aerofoils shows a plateau in the laminar BL region followed by a steep pressure increase. The pressure coefficient upstream the L-T transition point is lower over the modified aerofoils and then it becomes higher downstream the L-T transition. The pressure plateau shrinks increasing the WLE amplitude because it starts more downstream due to the amplitude increase and it finishes more upstream due to the L-T transition point movement. The laminar-turbulent transition point moves upstream when the amplitude is increased because the flow distortion at the LE is enhanced; this means that the disturbances generated at the LE are higher when the wavy amplitude is increased and this brings to an earlier L-T transition.

The effect of pressure distribution over the lift coefficient is shown in Figure 5 where the relative change between SLE and WLE cases in terms of piecewise distribution of lift is illustrated for the three modified aerofoils. The gradient form is intended to show how much contribution is available "per unit length" along the chord, which is a kind of normalisation. When integrated over the chord, it gives the total lift coefficient difference between SLE and WLE cases. The chord-wise interval $(\Delta x)$ is set to $2 h$ in order to match the first sampling point of WLE with that of SLE $(x=-0.5)$. Since three WLE amplitudes results are compared, the size of $\Delta x$ is defined by the largest wavy amplitude $(h=0.0250)$. The trend is consistent for the three wavy aerofoils, the piecewise distribution of lift is higher over the modified aerofoils between the end of the LE region and the L-T transition point. The main effect of the wavy amplitude can be observed in the LE region where an amplitude increase has a negative impact on the local lift. A second effect is the L-T transition point's upstream movement which shrinks the region of lift enhancement when the WLE amplitude is enhanced. Overall, the amplitude growth has a negative effect on the aerofoil lift but a small wavy amplitude (WLE1) allows to generate a higher lift than the straight counterpart.

The relative change between SLE and WLE cases in terms of piecewise distribution of pressure drag coefficient 
(Figure 6) shows also a consistent trend changing the WLE amplitude. The LE region has almost a negligible effect with low amplitude $(h=0.0075)$, while higher amplitudes tend to increase the LE region's drag with respect to the baseline case. The beneficial effect for the drag is observed downstream of the modified aerofoil L-T transition point. These results are consistent with previous studies 26 . The region of $\mathrm{L}-\mathrm{T}$ transition on the modified aerofoil has a negative contribution on drag coefficient but it decreases when the wavy amplitude is increased. The decrease of pressure drag coefficient with the amplitude growth, observed in Table III] is the result of these streamwise positive and negative effects. Overall, the amplitude increase has a positive effect on the pressure drag coefficient, even if the LE region shows the opposite trend. This suggests that LE flow distortion does not bring to a local benefit but it has a positive effect on BL development downstream and its interaction with the shock wave.

The skin friction drag increases with WLE amplitude growth (Table III). Figure 4 displays the time-averaged skin friction coefficient distribution over the upper surface of straight and wavy aerofoils and shows that the skin friction drag increase is located in the laminar BL region and is distributed over the aerofoil span. This can be explained with the LE flow distortion which modifies 2D laminar BL, essential to have a low skin friction drag on supercritical aerofoils. Figure 4 shows that the mean position of laminar separation is moved downstream by the wavy aerofoil with respect to the straight one. In particular, the trough section shows the most upstream separation and an additional small LSB close to the leading edge, while the peak section has the most downstream separation. This trend is consistent with different WLE amplitudes. The main difference between straight and modified aerofoils is that the first one shows laminar separation, reattachment, and then L-T transition, while the second one shows laminar separation and then L-T transition.

The mean aerodynamic analysis shows that modified aerofoils are able to increase the aerofoil efficiency over the range of analysed amplitudes. The best performance is obtained with $h=0.0075$, where the lift coefficient is increased and the drag coefficient is decreased with respect to the straight counterpart. Since an aerofoil in transonic flow at moderate angles of attack is characterised by flow unsteadiness, an analysis of flow oscillations over the aerofoils is essential to define WLEs aerodynamic performance.

\begin{tabular}{cccccc}
\hline & $C_{L}$ & $C_{D}$ & $C_{D p}$ & $C_{D f}$ & $C_{L} / C_{D}$ \\
\hline SLE & 0.943 & 0.084 & 0.081 & 0.00314 & 11.20 \\
WLE1 $(h=0.0075)$ & 1.003 & 0.074 & 0.070 & 0.00382 & 13.56 \\
WLE2 $(h=0.0125)$ & 0.944 & 0.073 & 0.069 & 0.00383 & 12.91 \\
WLE3 $(h=0.0250)$ & 0.883 & 0.072 & 0.067 & 0.00460 & 12.34 \\
\hline
\end{tabular}

TABLE III: Mean aerodynamic performance of wavy and straight aerofoils at $\alpha=7.0^{\circ}$.

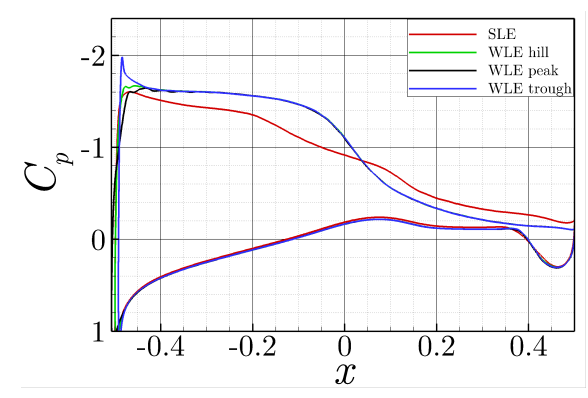

(a)

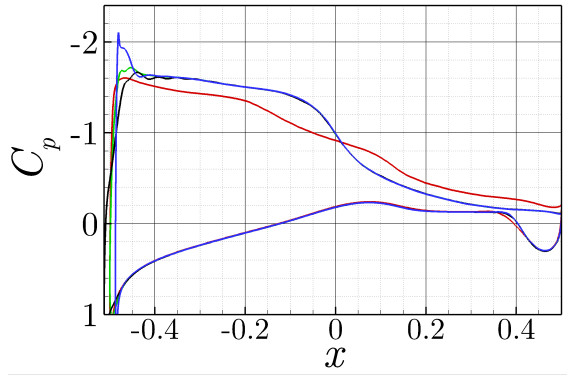

(b)

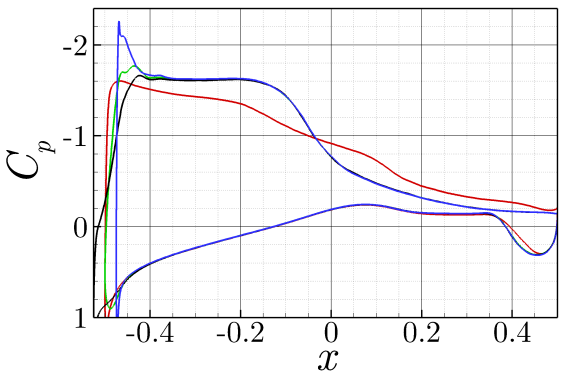

(c)

FIG. 3: Mean pressure coefficient distribution at WLE peak, hill and trough and over SLE at $\alpha=7.0^{\circ}$ for WLE1 (a), WLE2 (b) and WLE3 (c) aerofoils.

\section{B. Spanwise aerodynamic performance details}

The spanwise aerodynamic performance of a wavy aerofoil in transonic flows has been analysed in previous works ${ }^{26}$. The objective of this section is to extend the previous analysis to a higher incidence angle and to the influence of wavy amplitudes.

The mean lift coefficient distribution shows again two maxima at the peak and the trough of wavy geometry, where the first one is lower than the second one (Figure 7a). The lowest value is obtained at the hill section because the 


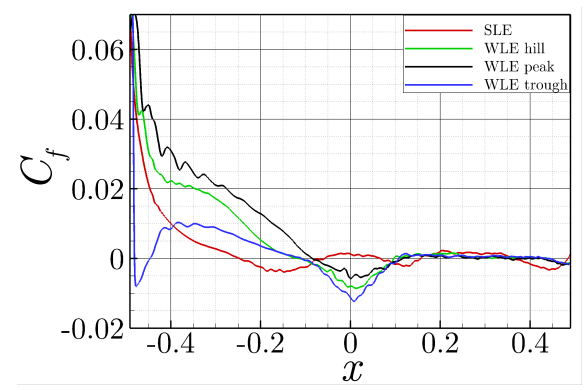

(a)

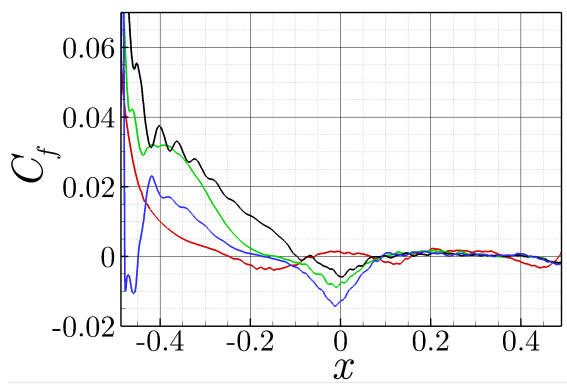

(b)

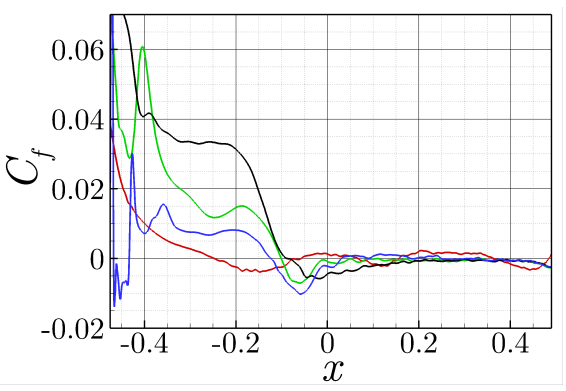

(c)

FIG. 4: Mean skin friction coefficient distribution over the suction side at WLE peak, hill and trough and over SLE at $\alpha=7.0^{\circ}$ for WLE1 (a), WLE2 (b) and WLE3 (c) aerofoils.

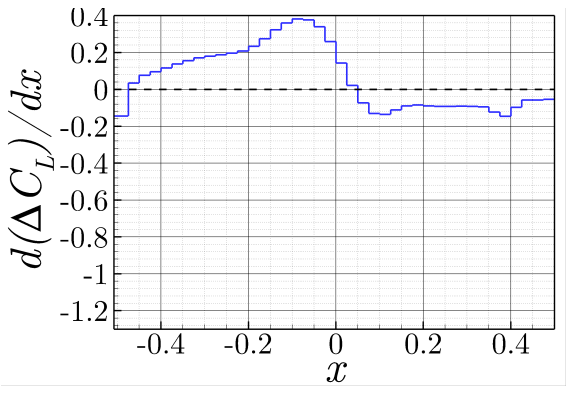

(a)



(b)



(c)

FIG. 5: Relative change in aerodynamic forces between SLE and WLE cases in terms of piecewise distribution of lift along the chord calculated from wall pressure $\left(d\left(\Delta C_{L}\right) / d x\right)$ for WLE1 (a), WLE2 (b) and WLE3 (c) aerofoils. The relative difference between SLE and WLE cases are denoted by $\Delta\{\}=.\{.\}_{W L E}-\{.\}_{S L E}$.

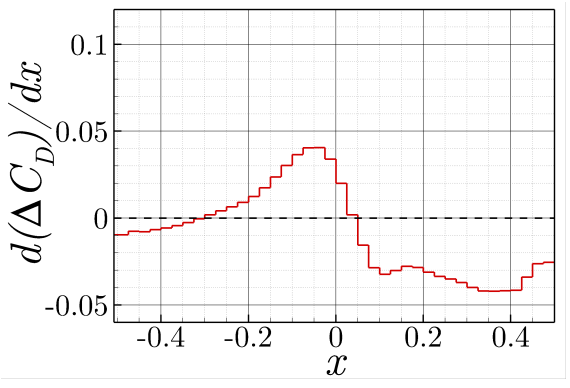

(a)

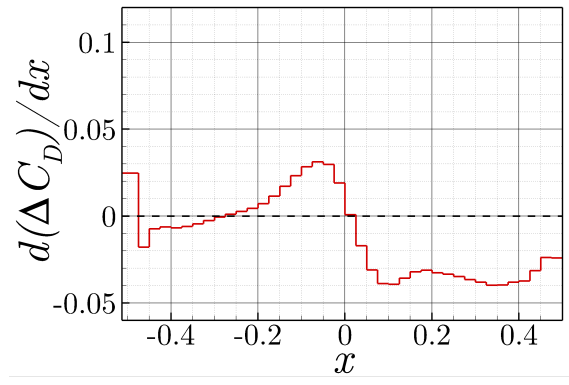

(b)



(c)

FIG. 6: Relative change in aerodynamic forces between SLE and WLE cases in terms of piecewise distribution of pressure drag along the chord calculated from wall pressure $\left(d\left(\Delta C_{D}\right) / d x\right)$ for WLE1 (a), WLE2 (b) and WLE3 (c) aerofoils. The relative difference between SLE and WLE cases are denoted by $\Delta\{\}=.\{.\}_{W L E}-\{.\}_{S L E}$.

derivative of the surface with respect to the $z$-direction has the highest value at the hill, thus the force has the largest spanwise component. The trough generates a lift higher than the peak because of the pressure distribution close to the leading edge. Increasing the wavy amplitude, the two maxima grow.

The drag coefficient shows a minimum at the trough and a maximum at the peak (Figure $7 \mathrm{~b}$ ). Their amplitude also increases when the wavy amplitude is enhanced. This spanwise distribution can be explained taking into account the pressure distribution close to the LE. The peak section is the one where there is the most gradual pressure decrease between the stagnation point and the pressure coefficient peak. This extended high-pressure region increases the drag. The opposite behaviour is observed at the trough; the pressure coefficient changes suddenly from the highest to lowest values of the pressure coefficient (Figure 3).

The resulting spanwise efficiency has a maximum at the trough and a minimum at the peak (Figure $7 \mathrm{c}$ ). The 
efficiency is evenly distributed between the maximum and the minimum at low wavy amplitudes $(h=0.0075)$. Increasing the wavy amplitude to high values $(h=0.0250)$, the efficiency in the trough region shows the maximum increasing quickly and the shape of the distribution becoming sharp, while in the peak region the minimum decreases slowly and the shape becomes quite flat. This is consistent with the overall decreased efficiency when WLE amplitude is increased (Table III).

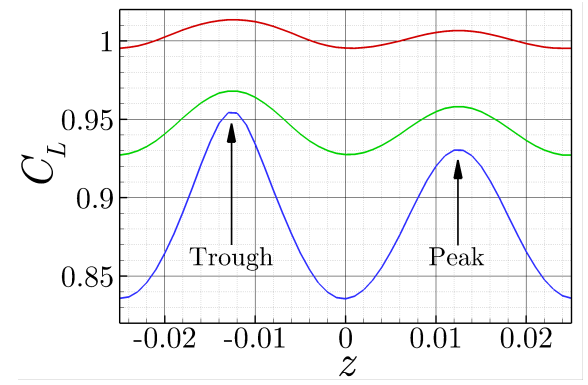

(a)

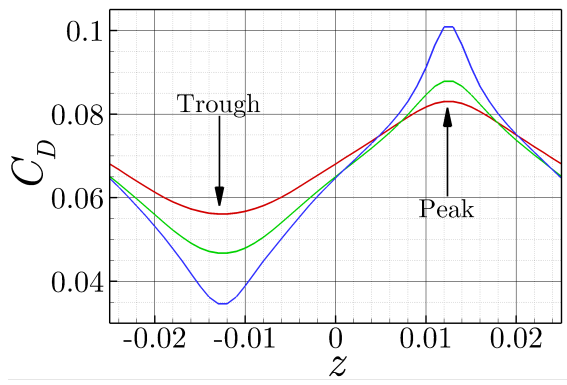

(b)



(c)

FIG. 7: Mean lift (a), drag (b), and efficiency (c) spanwise distribution over WLE1, WLE2 and WLE3 aerofoils at $\alpha=7.0^{\circ}$.

\section{The effect of $h_{L E}$ on time-averaged performance}

The time-averaged aerodynamic performance analysis has shown that WLE1 has the highest efficiency in this study. This result raises a question, whether an even smaller WLE amplitude can further improve the efficiency, or lift and drag become worse and tend to SLE case. Another simulation is needed to properly answer this question. The objective of this section is to investigate the WLE1 aerofoil in detail and compare the flow field with the baseline case. There are two possible explanations for WLE1 aerofoil's performance, the flow speed in the leading edge region or the streamwise vorticity generated by the flow distortion at the LE.

WLE1 aerofoil has still a crucial contribution to the flow speed up at the LE. The time-averaged speed up is higher both at the trough and at the hill with respect to the SLE case and this high-speed flow region has a positive effect on the peak section downstream $x=-0.45$ (Figure 8p). The overall higher speed up in the LE region is the main explanation of the WLE1 performance, while the streamwise vorticity is small and plays a marginal role. The flow field in the leading edge region needs to be investigated in order to understand the observed higher acceleration at the trough.

The laminar separation bubble generated in the trough region plays a key role in flow field acceleration. This can be observed in Figure 8 $8 \mathrm{a}, \mathrm{b}$ where the Mach number contour is displayed and the sonic line is highlighted with the black line. The pressure coefficient peak is in the region where the LSB is generated. Thus, the flow has a higher acceleration on WLE1 because the trough section has an effective higher geometric curvature at the LE due to the LSB. The BL in the leading edge region of both WLE1 and SLE aerofoils is further investigated. Figure 8r,d illustrates the pressure field and highlights the LSB canopy. The baseline aerofoil does not present a LSB in the leading edge region. The hypothesis is that once the WLE amplitude is sufficiently small to prevent a LSB from appearing, the WLE case would resemble the SLE counterpart.

\section{UNSTEADY CHARACTERISTICS}

The mean aerodynamic characteristics analysis shows a performance improvement of the modified aerofoil using different WLE amplitudes, but the control of unsteady phenomena is the main focus at moderate and high angles of attack. First of all, the aerodynamic force fluctuations are analysed to give an overview of the unsteady aerodynamic performance and the influence of wavy amplitude on mitigation of flow unsteadiness. Then, the unsteady motion of SLE and WLE aerofoils is studied in details showing the flow characteristics and identifying the different phenomena with a frequency filtering technique. 

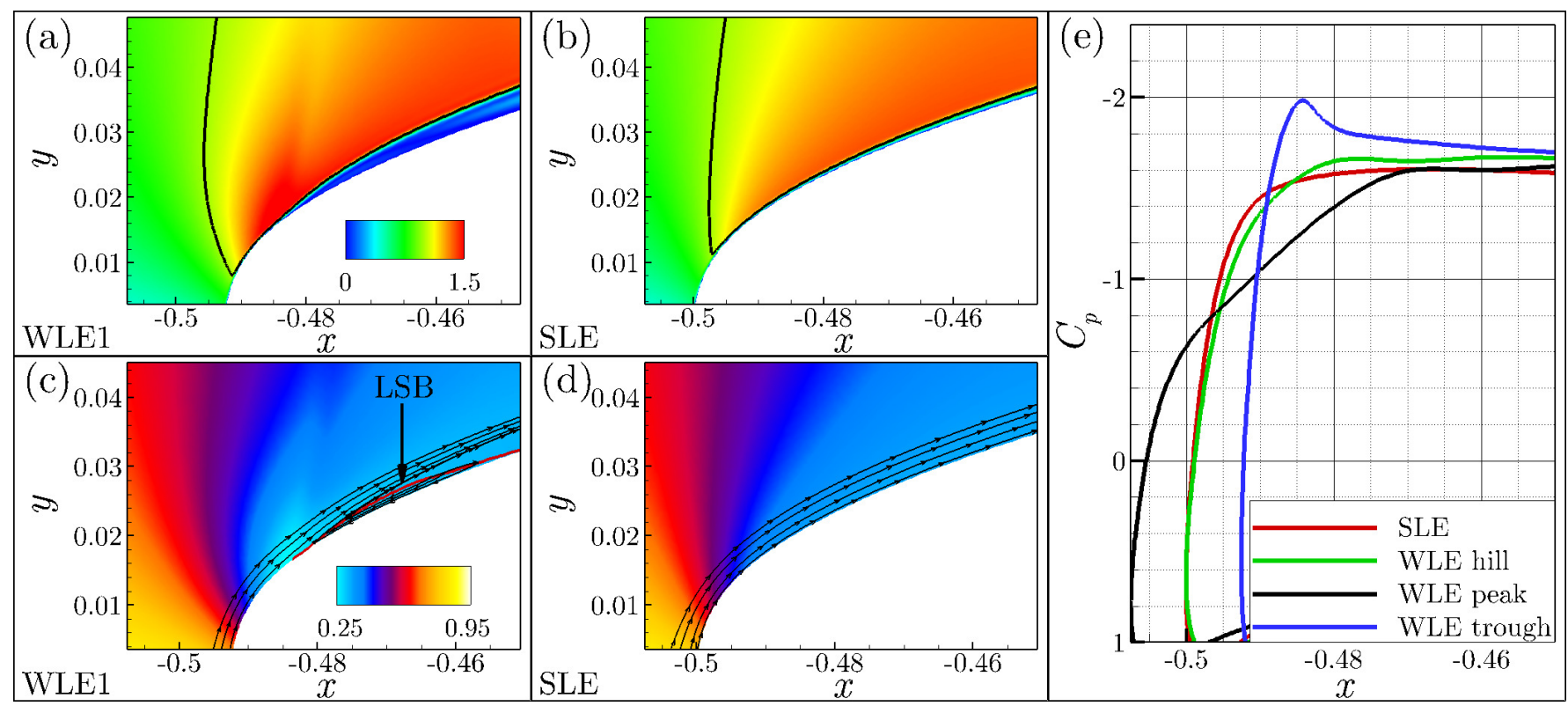

FIG. 8: Comparison of LE flow field at WLE1 trough (a,c) and over SLE (b,d). Mach number contour highlighting the sonic line (black line) (a,b). Pressure contour highlighting the LSB canopy (red line) and the streamlines close to the wall $(\mathrm{c}, \mathrm{d})$. Mean pressure coefficient distribution in the LE region at WLE1 peak, hill and trough and over SLE

(e).

\section{A. Aerodynamic force fluctuations}

Figure 9 illustrates the lift and drag coefficient time signal for baseline and modified aerofoils. The baseline case shows large low-frequency lift oscillations as the main unsteady phenomenon (Figure $9 \mathrm{a}$ ), which are consistent with previous studies $^{27}$ on the same aerofoil at higher Reynolds numbers. Consistent behaviour is observed for the drag coefficient time signal (Figure 9b). The power spectral density (PSD) displays two maxima (Figure 10), a low-frequency one $(S t=0.12)$ which might be related to shock buffet and a high-frequency one $(S t=1.2)$ which seems to be associated with vortex shedding 27 .

On the contrary, the modified aerofoils show different time signals changing the WLE amplitude. WLE1 aerofoil $(h=0.0075)$ presents two main lift and drag oscillations, a low-frequency one at $S t=0.1$ and a high-frequency one at $S t=0.9$ (Figure 10). The PSD shows mitigation of the low-frequency phenomenon with respect to the baseline case, while the high-frequency one increases its strength. The nature of these unsteady phenomena needs to be investigated in the following sections. The PSD for WLE2 aerofoil $(h=0.0125)$ shows that the low-frequency oscillation $(S t=0.14)$ is drastically decreased and the high-frequency one $(S t=0.64)$ becomes dominant for both lift and drag. At the highest amplitude considered in this study $(h=0.0250)$ the signals change again significantly. The low-frequency oscillation $(S t=0.08)$ is dominant and it is the strongest on the modified aerofoils, while the high-frequency component $(S t=1.0)$ is even lower than the one on the straight counterpart.

The main outcome of this unsteady performance analysis is that the leading edge waviness is able to suppress a strong low-frequency oscillation, generating a high-frequency one. Moreover, the wavy aerofoils' time signal shows always two components and their magnitude changes with different WLE amplitudes. The Strouhal number of maxima are summarised in Table IV] The low-frequency maximum initially decreases when WLE amplitude is enhanced and then it increases again. The high-frequency maximum has an opposite trend, it initially increases when the WLE amplitude is increased and then it decreases. It seems that the strength of the high-frequency oscillation has an influence on the strength of the low-frequency one. This hypothesis can be validated once the details of the unsteady phenomena are fully understood. The objective of the next sections is to characterise the phenomena over the baseline aerofoil, already observed in previous studies, and understand the phenomena over the modified aerofoils. 


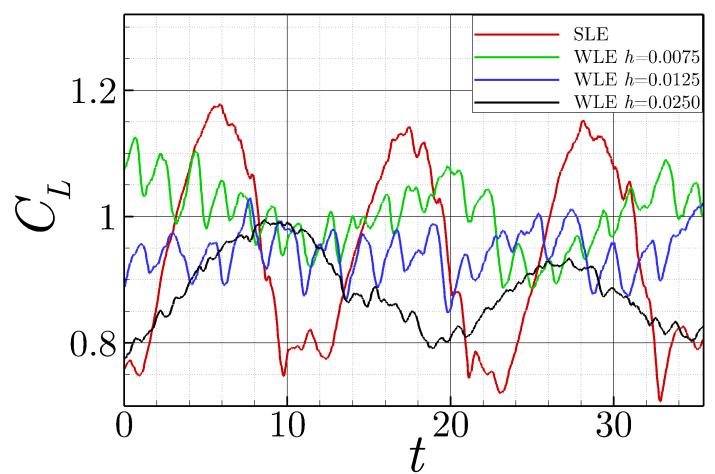

(a)



(b)

FIG. 9: Lift (a) and drag (b) coefficient time signal for SLE aerofoil and WLE1, WLE2 and WLE3 aerofoils.



(a)

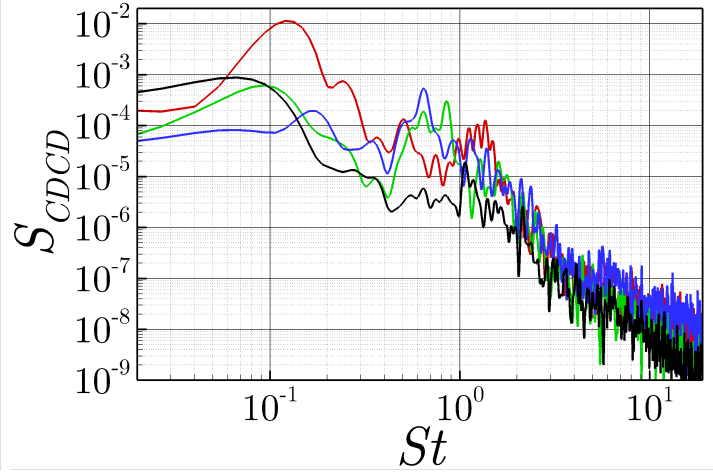

(b)

FIG. 10: Lift (a) and drag (b) coefficient power spectral density with respect to the Strouhal number for SLE aerofoil and WLE1, WLE2 and WLE3 aerofoils.

\begin{tabular}{ccc}
\hline & $I$ & $I I$ \\
\hline SLE & 0.12 & 1.20 \\
WLE1 $(h=0.0075)$ & 0.10 & 0.90 \\
WLE2 $(h=0.0125)$ & 0.14 & 0.64 \\
WLE3 $(h=0.0250)$ & 0.08 & 1.00
\end{tabular}

TABLE IV: Strouhal number of aerodynamic coefficients PSD maxima for SLE aerofoil and WLE1, WLE2 and WLE3 aerofoils.

\section{B. Overview of the unsteady motion}

The mitigation of aerodynamic coefficients oscillations shows that leading edge waviness is a promising passive technique for the control of a low-frequency oscillation which seems related to the shock buffet phenomenon. The analysis of the flow field over the aerofoils has the objective to understand the different phenomena. The main focus is the comparison between baseline and WLE2 aerofoils. The main reason is that the best result in terms of low-frequency oscillation control is obtained with $h=0.0125$. Secondly, the results shown in the previous section display always two maxima in the PSD of the wavy aerofoils which change their magnitude varying the amplitude, so it is assumed that the physical phenomena generating these maxima are the same for the different modified aerofoils. In addition, the characteristics of low-frequency oscillations over wavy aerofoils are compared with the ones over the baseline case.

Figure 11 shows the pressure gradient magnitude around the straight aerofoil during a full low-frequency cycle, which can be identified with the shock buffet phenomenon. Initially, the shock wave is at the most upstream position and it generates a large separation of turbulent BL (Figure 11 a). Pressure waves generated from vortices passing through the trailing edge decrease their strength because of BL separation. As a consequence, the turbulent boundary layer 
re-attaches, becomes thinner and the shock wave starts moving downstream (Figure 11p). At the most downstream position, there is a complex shock pattern because a $\lambda$-shape shock is followed by a normal shock (Figure 11:). The upstream shock of the $\lambda$ structure interacts with the laminar BL and it tends to separate it, generating a positive pressure gradient. Due to this strong interaction with the BL, there is a large separation which moves the transition point and large vortices develop downstream the SBLI region (Figure 11 $\mathrm{d}$ ). Strong sound waves are emitted from the TE and both turbulent BL and shock wave location move upstream. The shock wave reaches the most upstream position and the cycle starts again.

Unsteady phenomena over the baseline aerofoil are further investigated considering wall pressure signals at different locations on the upper surface (Figure 12). The low-frequency peak is observed everywhere on the upper surface but the PSD shows high values close to the TE where large vortical structures are observed and at the most upstream and downstream positions of the SBLI. The high-frequency peak shows a different trend, it is observed very close to the trailing edge and it reaches its highest value exactly at the TE, suggesting a relation with the vortex shedding phenomenon. Further investigations are required.

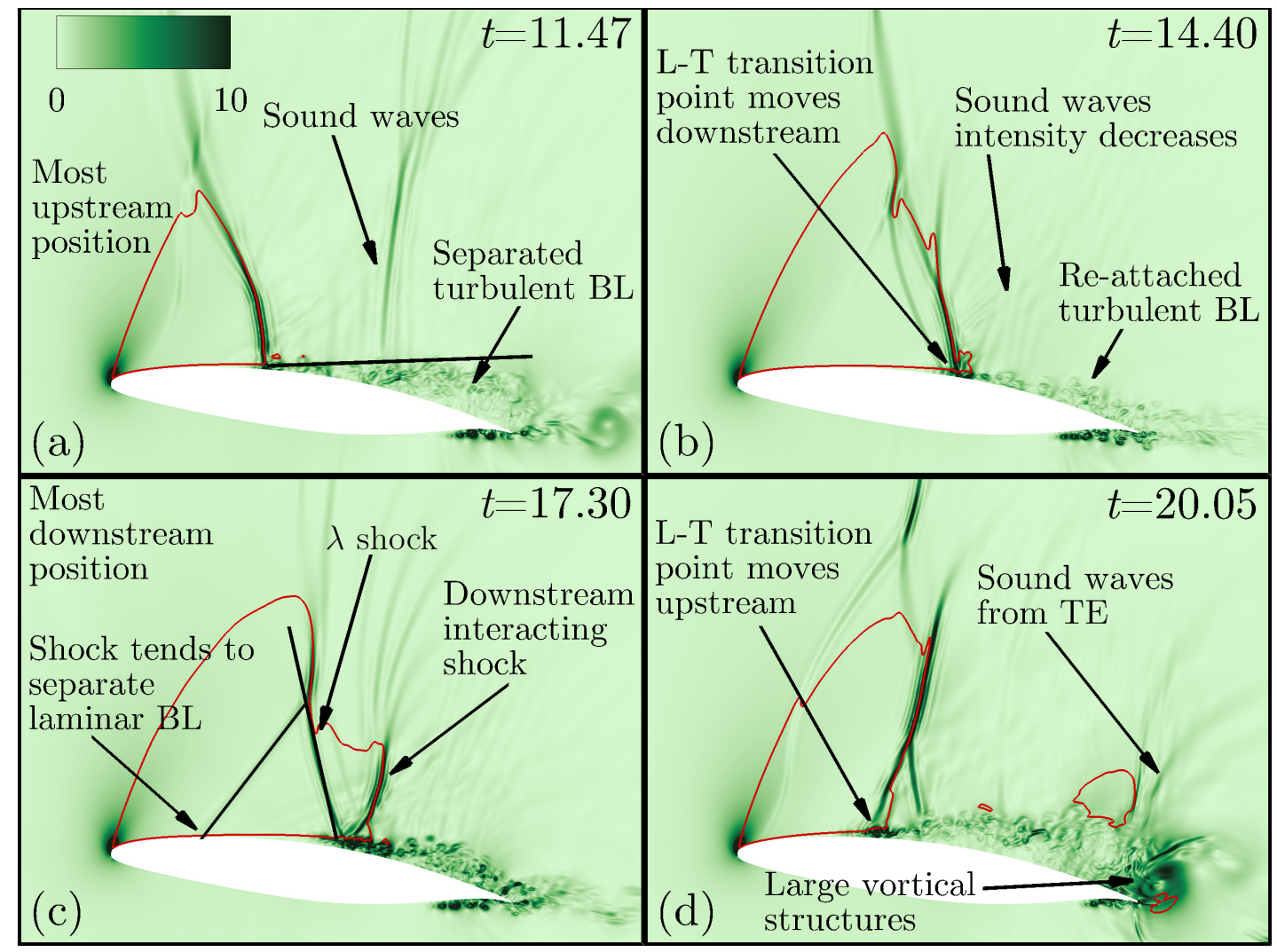

FIG. 11: Pressure gradient magnitude flow field around SLE aerofoil during one cycle of the shock buffet oscillation: most upstream position (a), shock wave downstream movement (b), most downstream position (c) and shock wave upstream movement $(\mathrm{d})$.

Figure 13 shows the pressure gradient magnitude around WLE3 aerofoil during a full cycle of the shock buffet oscillation. This permits to compare the characteristics of shock buffet phenomenon over the modified aerofoil with the ones over the straight counterpart. At the most upstream position, the turbulent boundary layer is separated and a $\lambda$-shape shock is observed (Figure $13 \mathrm{z}$ ). Once the BL re-attaches, the shock moves downstream up to the most downstream position (Figure 13b) where a $\lambda$-shape shock is observed again (Figure 13p). The interaction between $\lambda$-shape shock and laminar boundary layer tends to separate the BL and it is responsible for the shock wave upstream movement. As a consequence, large vortical structures develop downstream (Figure 13 d).

One of the main differences between baseline and wavy aerofoils is the amplitude of shock wave oscillation. Table $\mathrm{V}$ illustrates the details of shock positions, where $x_{S U}$ is the shock wave most upstream position, $x_{D U}$ is the most downstream position and $\Delta x_{S}$ is the oscillation's amplitude. WLE2 aerofoil is not reported because a shock oscillation is not observed. The overall result is that the shock wave oscillation over wavy aerofoils shows a lower amplitude than the straight counterpart. In particular, the shock wave displacement over WLE1 is about $45 \%$ of the one over SLE and over WLE3 is about 18\% of the one over SLE (Table V). WLE amplitude has an influence on the strength of 


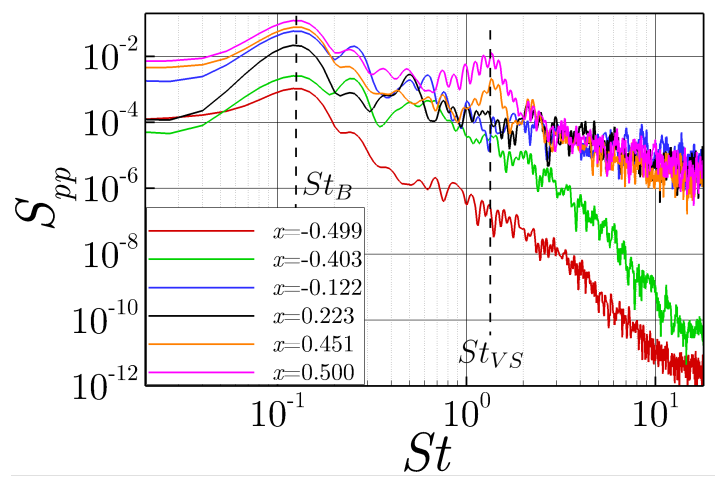

(a)

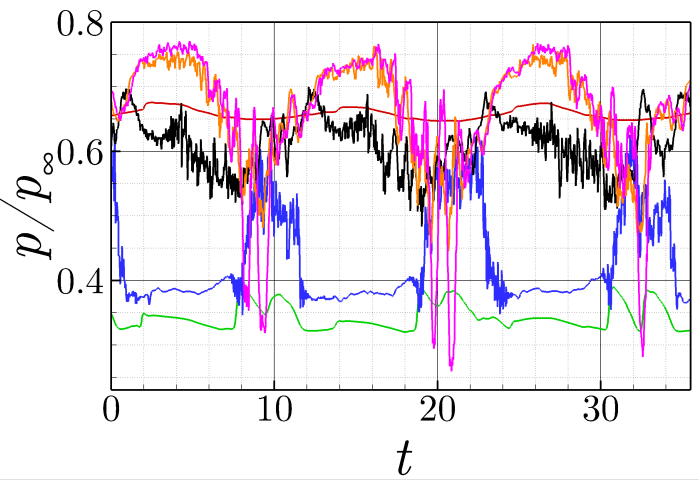

(b)

FIG. 12: Power spectral density with respect to the Strouhal number (a) and time signal (b) of wall pressure at different chordwise locations over the upper surface of SLE aerofoil.

oscillations, as it was observed at the beginning of this section. The shock wave has a larger oscillation over WLE3 where the high-frequency oscillation is weaker. This suggests a strong connection between low and high-frequency oscillations and requires a more detailed investigation of the high-frequency phenomenon. The consequences of shock oscillation lower amplitude are the generation of smaller vortical structures during the upstream movement and the emission of weaker pressure waves from TE and turbulent BL. Another major difference is the SBLI at the most downstream position. The baseline aerofoil shows a complex shock structure that interacts with the BL and tends to separate it, while the modified aerofoils display a $\lambda$-shape shock where the two shock waves of the $\lambda$ structure are very close. In addition, wavy leading edges create streamwise vorticity in the laminar BL which makes it more energetic, as it was observed in previous studies 26 . The shock structures together with the three dimensional laminar BL are the main flow characteristics which change the shock buffet phenomenon between straight and wavy aerofoils.

\begin{tabular}{cccc}
\hline & SLE & WLE1 & WLE3 \\
\hline$x_{S U}$ & -0.12 & 0.00 & -0.06 \\
$x_{S D}$ & 0.10 & 0.04 & 0.04 \\
$\Delta x_{S}$ & 0.22 & 0.04 & 0.10 \\
\hline
\end{tabular}

TABLE V: Shock wave oscillation details for baseline aerofoil and WLE1 and WLE3 aerofoils.

Figure 14 illustrates the wall pressure signals along the chord of WLE2 aerofoil, from trough to TE and its power spectral density with respect to the Strouhal number. The first interesting result is that the low-frequency peak is observed only around $x=0.1$ for both sections. The reason is that it is not observed a large separation as in the straight counterpart, the shock wave shows very limited upstream-downstream movements. The high-frequency pressure peak is observed on different chordwise locations for both sections, in particular, it reaches the highest value around the SBLI and the trailing edge. This shows the first difference between high-frequency phenomena on straight and wavy aerofoils. The high-frequency phenomenon on SLE aerofoil is detected only close to the TE, while the one on WLE2 aerofoil can be observed on different locations of the upper surface. Additional local unsteady phenomena can be observed from this analysis. First of all, a bump of wall pressure PSD is detected at $x=0.118$ around $S t=5$ which disappears at the TE. Secondly, a bump appears also at $S t=11$ close to the leading edge. The latter seems to be related to Kelvin-Helmholtz instability due to the LSB observed at the trough in Figure 4 These phenomena do not appear at the other sections and in aerodynamic coefficients PSD, thus they do not have an influence on unsteady aerodynamic performance. Figure 15 shows the pressure gradient magnitude around WLE2 aerofoil during a full high-frequency cycle, at the minimum and maximum values. The flow field does not display clearly the driving mechanism of this unsteady oscillation. At the lift minimum, there is a BL thickening in the L-T transition region, this bends the $\lambda$-shape shock and the forward shock moves upstream (Figure 15a). At the lift maximum the BL thins and the $\lambda$-shape shock moves in the opposite direction (Figure 15p). It is observed downstream the advection of vortical structures, generated from BL thickening and thinning. The high-frequency phenomenon seems to be driven by BL oscillations in L-T transition region, but further investigations are needed. 


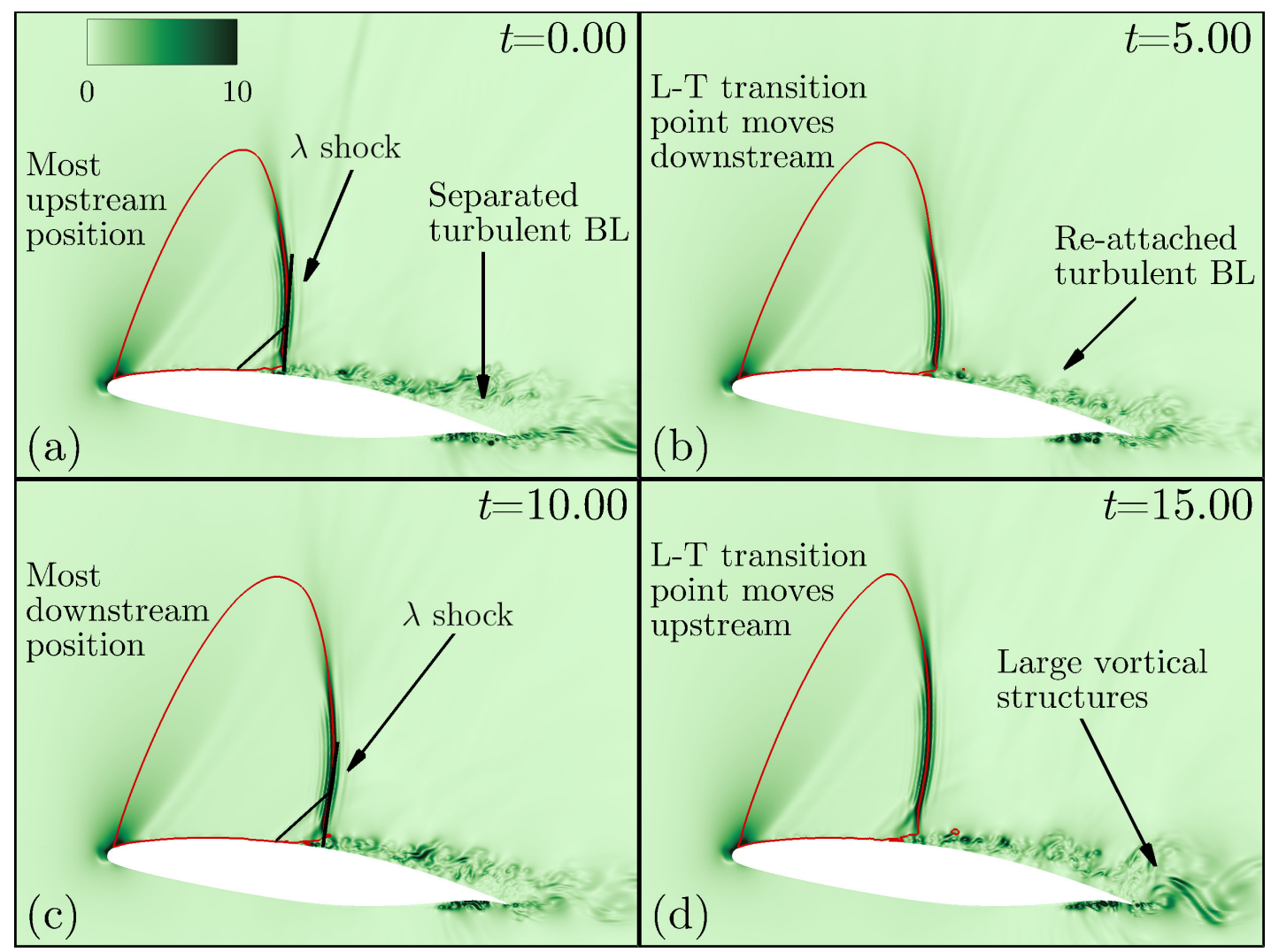

FIG. 13: Pressure gradient magnitude flow field at the hill section of WLE3 aerofoil during one cycle of the shock buffet oscillation: most upstream position (a), shock wave downstream movement (b), most downstream position (c) and shock wave upstream movement (d).



(a)



(b)

FIG. 14: Power spectral density with respect to the Strouhal number (a) and time signal (b) of wall pressure at different chordwise locations over the upper surface of WLE2 trough section.

\section{Physical phenomena identification and analysis}

Previous studies have used different techniques to analyse unsteady phenomena and to identify coherent structures. Proper Orthogonal Decomposition (POD) searches for the most energetic modes whereby coherent structures with high energy content are likely to be represented by POD basis functions 38 . Dynamic Mode Decomposition (DMD) targets the coherent structures' temporal periodicity and distinguishes modes with respect to their linear amplification 39 . These methods fail when relevant coherent structures occur at low energies or at multiple frequencies. Spectral Proper Orthogonal Decomposition (SPOD) has been developed to overcome this limitation and enables a clear separation of 


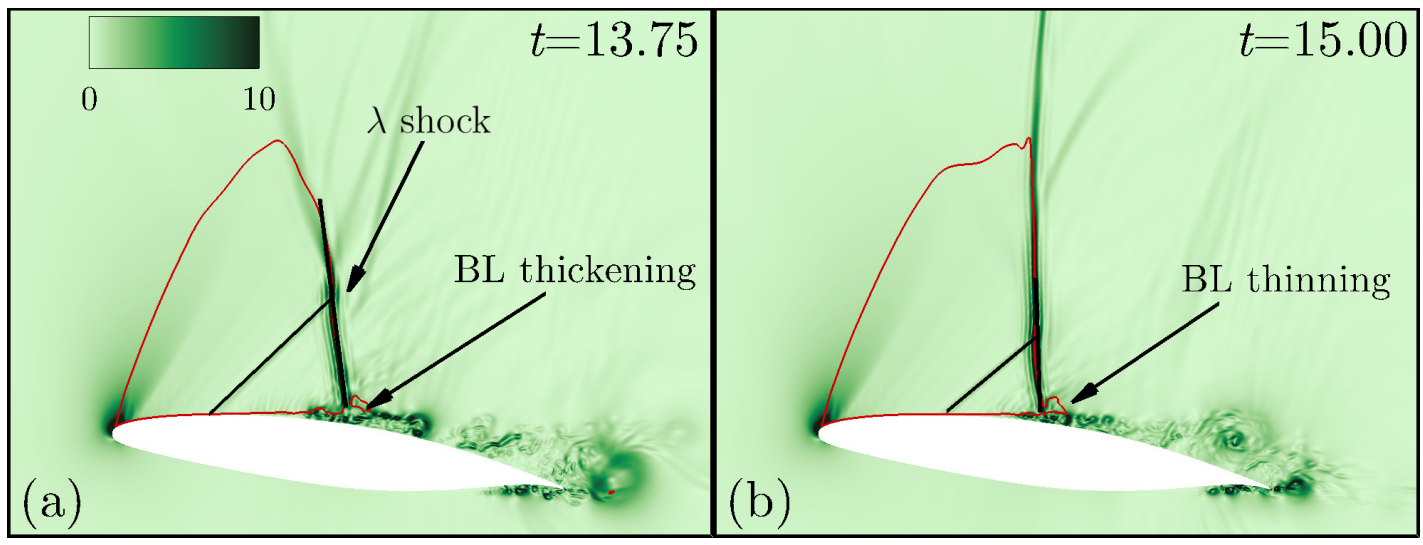

FIG. 15: Pressure gradient magnitude flow field at the hill section of WLE2 aerofoil at the high frequency oscillation's minimum (a) and maximum (b).

phenomena that occur at multiple frequencies and energies 40 .

In this study, a different approach is used based on a frequency filtering of the flow 14 . The advantage is that once the flow field is filtered, it is still possible to analyse its evolution over time and results are not limited to a contour of the modes. The limitation is that results do not include an energetic representation of the modes like with SPOD. The details of the method are given as follows.

- The Fourier transform of an arbitrary field variable is evaluated for every grid point.

- Real and imaginary parts are multiplied by a weighting function

$$
g(f)=\exp \left[-a\left(f-f_{0}\right)^{2}\right]+\exp \left[-a\left(f-f_{0}\right)^{2}\right]
$$

where $f_{0}$ is the frequency of the unsteady phenomenon and $a$ is an arbitrary parameter that defines the function's wideness. Increasing the value of $a$, the effect of close frequencies is filtered out.

- The inverse Fourier transform is evaluated for every grid point.

The result is a filtered field of the initial field variable. Figure 16 shows an example of the original and filtered spanwise vorticity flow field on WLE2 aerofoil. The high magnitude of the filtered field indicates where the field is more energetic at the given frequency, thus the location of the oscillation's origin and this permits to understand the unsteady phenomenon.
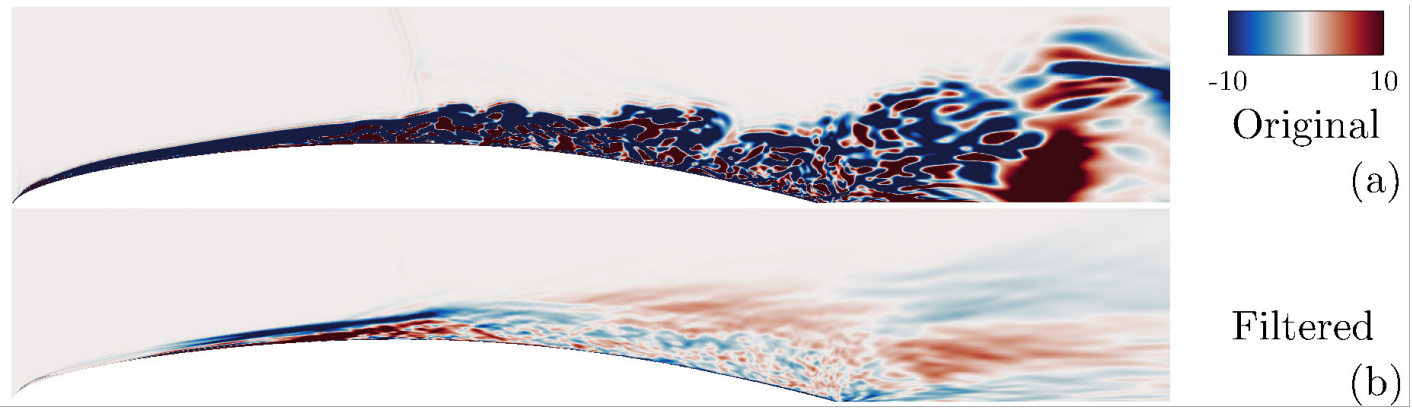

(a)

\section{Filtered}

FIG. 16: Instantaneous original (a) and filtered (b) spanwise vorticity at the trough section of WLE2 aerofoil.

The objective of this section is to exploit the frequency filtering method to analyse the unsteady phenomena on both straight and wavy aerofoils. It was observed at the beginning of this section that each aerofoil shows two main unsteady phenomena and their frequencies are summarised in Table IV It is clear that the low-frequency peak (first column of Table IV is caused by shock buffet oscillation, thus the main focus is the characterisation of the second peak's unsteadiness.

The high-frequency oscillation on SLE aerofoil is detected at the trailing edge. Since Figure 11 displays large vortical structures in the TE region, the pressure coefficient field is filtered. Figure 17 shows the filtered field around the TE 
during one cycle of the high-frequency oscillation. The circular structures observed at the trailing edge suggest a periodic release of vortices from the aerofoil. Thus, this unsteady oscillation can be identified with vortex shedding. The vortex shedding phenomenon on a circular cylinder is usually detected at $S t \simeq 0.2$. The Strouhal number on an aerofoil is calculated with respect to the chord, while on a cylinder it is defined with respect to the diameter. Since the Strouhal number obtained in this case is 6 times larger, the vortex size is expected to be 6 times smaller than the aerofoil chord. A vortex diameter of about 0.16 is observed in Figure 11. This validation, together with the frequency filtering analysis, proves that the unsteady phenomenon at high frequency is vortex shedding. The shock wave is oscillating at low frequency and the SBLI point is moving backward and forward periodically over the upper surface of the aerofoil. During this oscillation, vortical structures are released periodically at a higher frequency causing the vortex shedding phenomenon. This means that the mitigation of this high-frequency phenomenon with active or passive control techniques can have a positive effect also on the shock buffet phenomenon. That's why previous approaches with passive techniques modifying TE geometry were successfully controlling shock buffet oscillation ${ }^{1}$. The problem is that those techniques were drastically decreasing aerofoil efficiency, while active techniques, which can be applied only at high angles of attack, show better results ${ }^{2}$.

The high-frequency phenomenon on WLE aerofoil has been already analysed in Figure 15. It has been observed a periodic oscillation in the SBLI region. For this reason, the spanwise vorticity field is filtered. The region highlighted by the filtered field is located in the LSB region up to the SBLI point (Figure 18). Figure 19 shows the streamwise velocity field in the BL region upstream SBLI. The black line defines the LSB canopy and it shows clearly a highfrequency BL thickening and thinning. This kind of phenomenon has been observed in previous studies ${ }^{42}$, it is related to LSB breathing and, as a consequence, it modifies the $\lambda$ shock shape as shown in Figure 15. It is possible to observe the same unsteadiness at the TE with a time lag with respect to the one in the laminar boundary layer (Figure (18). This means that the origin of the bubble breathing phenomenon is in the laminar BL, close to the laminar-turbulent transition point, and this generates vortical structures that are convected up to the TE. This explains why the highest values of high-frequency peaks in Figure 14 are close to L-T transition and to TE. WLE aerofoil modifies the flow at the leading edge and the downstream development of the flow in laminar BL is responsible for another unsteady phenomenon, crucial to control shock buffet oscillation. This permits to explain the bump observed at $x=0.118$ in Figure 14. In this region, small vortices of different sizes are generated and then convected downstream. The range of frequencies detected in Figure 14 is consistent with the size of the vortices displayed in Figure 15

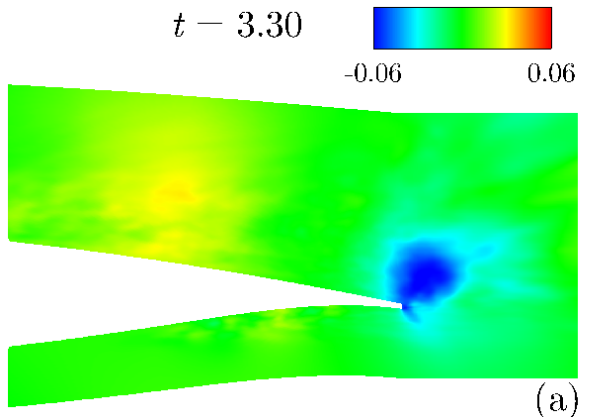

$t-3.90$

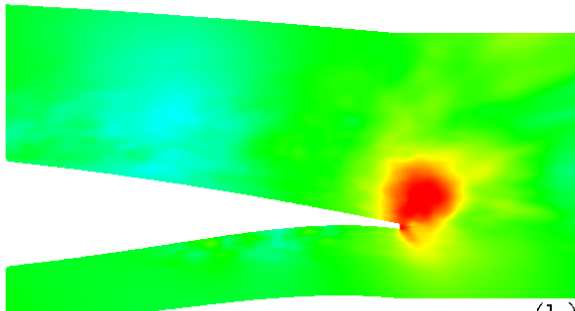

(a) $t-4.50$

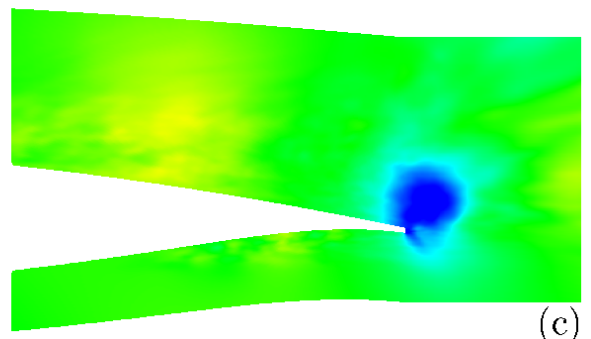

FIG. 17: Filtered pressure coefficient field around the trailing edge of SLE aerofoil during one cycle of the high frequency oscillation. Negative filtered pressure coefficient shows the release of a vortex from the trailing edge (a), followed by a region of positive filtered pressure coefficient (b), then the cycle starts again and another vortex is released (c).

\section{CONCLUDING REMARKS}

Wavy leading edges over supercritical aerofoils in transonic flows show promising results. This study has extended the work of a previous analysis 26 considering the effect of WLE amplitude on mean aerodynamic performance and on mitigation of shock buffet unsteady phenomenon.

In Section III the mean aerodynamic performance has been studied both in streamwise and spanwise directions together with the influence of wavy amplitude on aerofoil performance. The mean efficiency can be increased for a wide range of WLE amplitudes but the best performance is obtained with the lowest one $(h=0.0075)$. The results show that a small waviness is sufficient to create an important drag decrease. The main effect of WLE amplitude on lift coefficient is around the LE region which has a negative effect on lift compared to the SLE case. The effect becomes worse when WLE amplitude is enhanced. A second effect is the upstream movement of the L-T transition point which 




FIG. 18: Filtered spanwise vorticity field at the trough section of WLE2 aerofoil during one cycle of the high frequency oscillation. The minimum (a), maximum (b) and the following minimum (c) of the oscillation are shown.

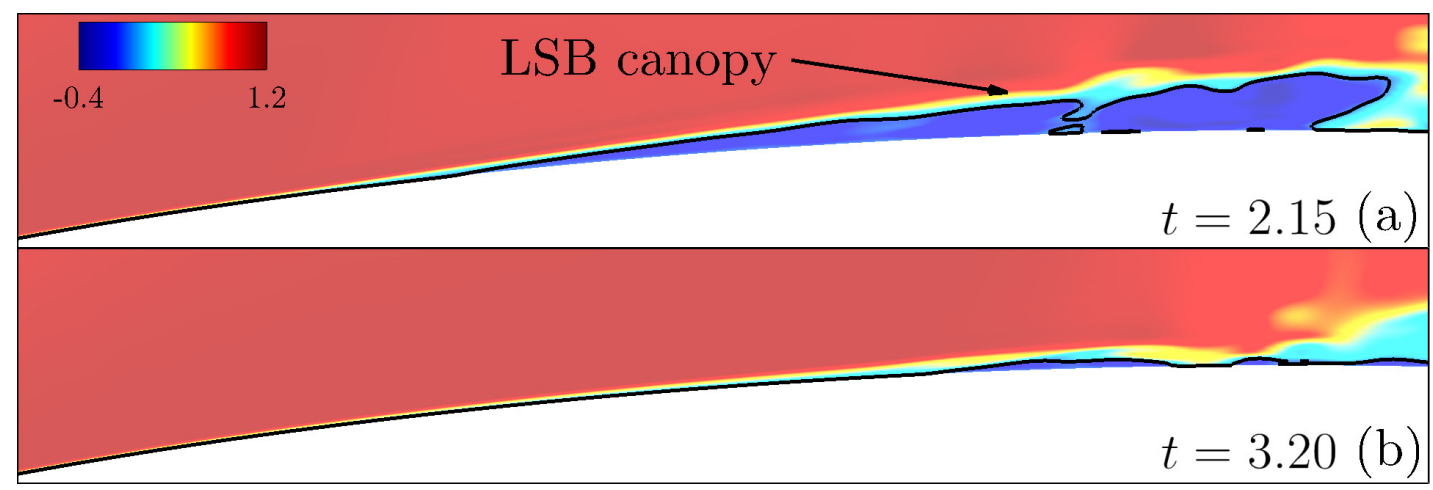

FIG. 19: Streamwise velocity field at the trough section of WLE2 aerofoil showing the minimum (a) and maximum (b) of the LSB breathing oscillation, related to periodic thickening and thinning of the LSB respectively. Black line highlights LSB canopy.

shrinks the region of lift enhancement when WLE amplitude is increased. The main effect of wavy amplitude on drag coefficient is in the LE region which has almost a negligible effect on drag at low amplitudes, while wavy amplitude growth tends to increase the drag with respect to the SLE case. The beneficial effect on drag is observed downstream of the modified aerofoil's L-T transition point.

Section IV has analysed the unsteady results in detail, starting from the shock buffet phenomenon and the effect of a wavy leading edge on it. Then, high-frequency phenomena have been identified and studied on both baseline and modified aerofoils. The main results are summarised as follows.

- Shock buffet phenomenon can be controlled by a wavy aerofoil and it changes its strength modifying the wavy amplitude. WLE aerofoils show a decrease in shock wave oscillation's amplitude. The strong interaction between shock wave and BL generates a large separation on SLE aerofoil, while this separation is not observed on the wavy counterpart. This difference during low-frequency oscillations is related to a more energetic BL on wavy aerofoils because of streamwise vorticity created upstream in the LE region.

- The high-frequency oscillation on baseline aerofoils is related to vortex shedding, while the one on modified aerofoils is related to LSB breathing. LSB breathing is responsible for a periodic thickening and thinning of the laminar BL upstream SBLI region. As a consequence, small vortices are generated and they convect downstream creating large vortical structures at the TE. It is observed a strong connection between shock buffet and LSB breathing phenomena on wavy aerofoils. When the shock buffet's strength is decreased, LSB breathing's strength is increased and vice versa.

Future works aim to extend the study on mean and unsteady analysis at $\alpha=7.0^{\circ}$ simulating two possible wavy wing configurations with a sweep angle. A WLE swept wing should be studied at the most efficient condition, which 
represents the cruise condition, and at the shock buffet condition. This allows to find the best configuration for a swept wing with wavy leading edges and it permits to analyse the mean and unsteady performance of WLE aerofoils with respect to SLE one in an aeronautical case.

\title{
ACKNOWLEDGEMENTS
}

The authors gratefully acknowledge the support of EPSRC (Engineering and Physical Sciences Research Council) for the present work under the CDT (Centre for Doctoral Training) in NGCM (Next Generation Computational Modelling) at the University of Southampton. We also acknowledge the high-performance computing facilities and services of the UK National Supercomputer ARCHER and the local IRIDIS5 at the University of Southampton in the completion of this work. We finally acknowledge the Dassault Systèmes for providing V2C supercritical profile.

\section{NOMENCLATURE}

\author{
AoA Angle of Attack \\ BL Boundary Layer \\ DNS Direct Numerical Simulation \\ ILES Implicit Large Eddy Simulation \\ LE Leading Edge \\ LES Large Eddy Simulation \\ LSB Laminar Separation Bubble \\ L-T Laminar-Turbulent \\ SBLI Shock wave Boundary Layer Interaction \\ SCB Shock Control Bump \\ SLE Straight Leading Edge \\ TE Trailing Edge \\ TED Trailing Edge Device \\ VG Vortex Generator \\ WLE Wavy Leading Edge \\ St Strouhal number
}

\section{DATA AVAILABILITY}

All data supporting this study are openly available from the University of Southampton repository at http://dx.doi.org/10.5258/SOTON/XXXXXX.

\footnotetext{
${ }^{1}$ M. Iovnovich and D. Raveh, "Transonic unsteady aerodynamics in the vicinity of shock-buffet instability," Journal of Fluids and Structures 29, 131-142 (2012).

${ }^{2}$ C. Gao, W. Zhang, and Z. Ye, "Numerical study on closed-loop control of transonic buffet suppression by trailing edge flap," Computers \& Fluids 132, 32-45 (2016).

${ }^{3}$ D. Caruana, A. Mignosi, M. Corrège, A. Le Pourhiet, and A. Rodde, "Buffet and buffeting control in transonic flow," Aerospace Science and Technology 9,605-616 (2005).

${ }^{4}$ M. Rybalko, H. Babinsky, and E. Loth, "Vortex generators for a normal shock/boundary layer interaction with a downstream diffuser," Journal of Propulsion and Power 28, 71-82 (2012).

${ }^{5}$ N. Titchener and H. Babinsky, "Shock wave/boundary-layer interaction control using a combination of vortex generators and bleed," AIAA journal 51, 1221-1233 (2013).

${ }^{6} \mathrm{H}$. A. Holden and H. Babinsky, "Effect of microvortex generators on seperated normal shock/boundary layer interactions," Journal of Aircraft 44, 170-174 (2007).

${ }^{7}$ J. Huang, Z. Xiao, J. Liu, and S. Fu, "Simulation of shock wave buffet and its suppression on an oat15a supercritical airfoil by iddes," Science China Physics, Mechanics and Astronomy 55, 260-271 (2012).

${ }^{8}$ P. Molton, J. Dandois, A. Lepage, V. Brunet, and R. Bur, "Control of buffet phenomenon on a transonic swept wing," AIAA journal 51, 761-772 (2013).

${ }^{9}$ G. Godard and M. Stanislas, "Control of a decelerating boundary layer. part 1: Optimization of passive vortex generators," Aerospace Science and Technology 10, 181-191 (2006).

${ }^{10}$ S. Koike, K. Nakakita, T. Nakajima, S. Koga, M. Sato, H. Kanda, K. Kusunose, M. Murayama, Y. Ito, and K. Yamamoto, "Experimental investigation of vortex generator effect on two-and three-dimensional nasa common research models," in 53rd AIAA Aerospace Sciences Meeting (2015) p. 1237.

${ }^{11}$ Y. Ito, K. Yamamoto, K. Kusunose, S. Koike, K. Nakakita, M. Murayama, and K. Tanaka, "Effect of vortex generators on transonic
} swept wings," Journal of Aircraft 53, 1890-1904 (2016). 
${ }^{12}$ E. Stanewsky, J. Délery, J. Fulker, and W. Geißler, EUROSHOCK-Drag Reduction by Passive Shock Control: Results of the Project EUROSHOCK, AER2-CT92-0049 Supported by the European Union, 1993-1995, Vol. 56 (Springer Science \& Business Media, 2013).

${ }^{13}$ J. Birkemeyer, H. Rosemann, and E. Stanewsky, "Shock control on a swept wing," Aerospace Science and Technology 4, 147-156 (2000).

${ }^{14}$ B. König, M. Pätzold, T. Lutz, E. Krämer, H. Rosemann, K. Richter, and H. Uhlemann, "Numerical and experimental validation of three-dimensional shock control bumps," Journal of Aircraft 46, 675-682 (2009).

${ }^{15}$ M. Pätzold, T. Lutz, E. Kramer, and S. Wagner, "Numerical optimization of finite shock control bumps," in 44th AIAA Aerospace Sciences Meeting and Exhibit (2006) p. 1054.

${ }^{16}$ J. P. Eastwood and J. P. Jarrett, "Toward designing with three-dimensional bumps for lift/drag improvement and buffet alleviation," AIAA journal 50, 2882-2898 (2012).

${ }^{17}$ R. Mayer, T. Lutz, E. Krämer, and J. Dandois, "Control of transonic buffet by shock control bumps on wing-body configuration," Journal of Aircraft 56, 556-568 (2019).

${ }^{18}$ P. Bruce and S. Colliss, "Review of research into shock control bumps," Shock Waves 25, 451-471 (2015).

${ }^{19}$ S. Bogdanski, K. Nübler, T. Lutz, and E. Krämer, "Numerical investigation of the influence of shock control bumps on the buffet characteristics of a transonic airfoil," in New results in numerical and experimental fluid mechanics IX (Springer, 2014 ) pp. 23-32.

${ }^{20}$ R. Pérez-Torró and J. W. Kim, "A large-eddy simulation on a deep-stalled aerofoil with a wavy leading edge," Journal of Fluid Mechanics 813, 23-52 (2017).

${ }^{21}$ K. L. Hansen, N. Rostamzadeh, R. M. Kelso, and B. B. Dally, "Evolution of the streamwise vortices generated between leading edge tubercles," Journal of Fluid Mechanics 788, 730-766 (2016).

${ }^{22}$ L. Siconolfi, S. Camarri, and J. H. Fransson, "Boundary layer stabilization using free-stream vortices," Journal of Fluid Mechanics 764 (2015).

${ }^{23}$ N. Rostamzadeh, K. Hansen, R. Kelso, and B. Dally, "The formation mechanism and impact of streamwise vortices on naca 0021 airfoil's performance with undulating leading edge modification," Physics of Fluids 26, 107101 (2014).

${ }^{24}$ D. Custodio, C. Henoch, and H. Johari, "Aerodynamic characteristics of finite span wings with leading-edge protuberances," AIAA journal 53, 1878-1893 (2015).

${ }^{25}$ H. Yoon, P. Hung, J. Jung, and M. Kim, "Effect of the wavy leading edge on hydrodynamic characteristics for flow around low aspect ratio wing," Computers \& Fluids 49, 276-289 (2011).

${ }^{26}$ E. Degregori and J. W. Kim, "An investigation on a supercritical aerofoil with a wavy leading edge in a transonic flow," Physics of Fluids 32, 076105 (2020).

${ }^{27}$ D. Szubert, I. Asproulias, F. Grossi, R. Duvigneau, Y. Hoarau, and M. Braza, "Numerical study of the turbulent transonic interaction and transition location effect involving optimisation around a supercritical aerofoil," European Journal of Mechanics-B/Fluids 55, 380-393 (2016).

${ }^{28}$ M. Zauner, N. De Tullio, and N. D. Sandham, "Direct numerical simulations of transonic flow around an airfoil at moderate reynolds numbers," AIAA Journal 57, 597-607 (2018).

${ }^{29}$ D. J. Garmann, M. R. Visbal, and P. D. Orkwis, "Comparative study of implicit and subgrid-scale model large-eddy simulation techniques for low-reynolds number airfoil applications," International Journal for Numerical Methods in Fluids 71, 1546-1565 (2013).

${ }^{30}$ J. W. Kim and P. J. Morris, "Computation of subsonic inviscid flow past a cone using high-order schemes," AIAA journal 40, 1961-1968 (2002).

${ }^{31}$ J. W. Kim, A. S. H. Lau, and N. D. Sandham, "Proposed boundary conditions for gust-airfoil interaction noise," AIAA journal 48, $2705-2710(2010)$.

32 J. W. Kim, "Optimised boundary compact finite difference schemes for computational aeroacoustics," Journal of Computational Physics 225, 995-1019 (2007).

${ }^{33}$ J. W. Kim, "High-order compact filters with variable cut-off wavenumber and stable boundary treatment," Computers \& Fluids 39, 1168-1182 (2010).

${ }^{34}$ J. W. Kim and D. J. Lee, "Adaptive nonlinear artificial dissipation model for computational aeroacoustics," AIAA journal 39, 810-818 (2001).

${ }^{35}$ J. W. Kim and D. J. Lee, "Generalized characteristic boundary conditions for computational aeroacoustics," AIAA journal 38, 2040-2049 (2000).

${ }^{36}$ J. W. Kim and D. Joo, "Generalized characteristic boundary conditions for computational aeroacoustics, part 2," AIAA journal 42, $47-55(2004)$.

${ }^{37}$ J. W. Kim, "Quasi-disjoint pentadiagonal matrix systems for the parallelization of compact finite-difference schemes and filters," Journal of Computational Physics 241, 168-194 (2013).

${ }^{38}$ J. L. Lumley, "The structure of inhomogeneous turbulent flows," Atmospheric turbulence and radio wave propagation (1967).

${ }^{39}$ P. J. Schmid, "Dynamic mode decomposition of numerical and experimental data," Journal of fluid mechanics 656, 5-28 (2010).

${ }^{40}$ M. Sieber, C. O. Paschereit, and K. Oberleithner, "Spectral proper orthogonal decomposition," Journal of Fluid Mechanics 792, 798-828 (2016).

${ }^{41}$ J. M. Turner and J. W. Kim, "Trailing-edge noise generation from a flat-plate aerofoil interacting with a prescribed vortex," Journal of Sound and Vibration, 115654 (2020).

${ }^{42}$ J. Dandois, I. Mary, and V. Brion, "Large-eddy simulation of laminar transonic buffet," Journal of Fluid Mechanics 850, 156-178 (2018). 